methodology and study design, theoretical frameworks proposed and the impacts described in each report.

Results The majority of research was conducted in the Pacific or Caribbean region $(49 \%, 44 \%)$ and primarily focused on fishing and crop farming $(39 \%, 26 \%)$. The findings indicate that there is a predominance of research focusing on the environmental impact of marine and costal resources (mostly fishing), and very limited evidence regarding the impact of locally implemented food production programs on human health, particularly nutrition and diet-related outcomes. Furthermore, there was a general absence of explicit theoretical frameworks or logical models to explain how CFPIs may bring about health, social, economic or environmental change. The studies which reported the impacts on CFPIs tended to report the impact of management factors, social characteristics or higher level socio-political environment on CFPIs and subsequent food security.

Conclusion Evidence of the health and other impacts of CPFIs in SIDS is limited and the approaches taken inconsistent. This review demonstrates the need and provides a basis for developing a coherent body of methods to examine the impacts of CFPIs and provide evidence to guide policy.

\section{P5 EXPLORING CONTEXTUAL PREDICTORS AND MODIFIERS OF ASSOCIATIONS BETWEEN THE NEIGHBOURHOOD BUILT ENVIRONMENT AND OBESITY ACROSS THE UK}

${ }^{1} \mathrm{KE}$ Mason*, ${ }^{1} \mathrm{~N}$ Pearce, ${ }^{2} \mathrm{~S}$ Cummins. ${ }^{1}$ Non-Communicable Disease Epidemiology, London School of Hygiene and Tropical Medicine, London, UK; ${ }^{2}$ Public Health, Environments and Society, London School of Hygiene and Tropical Medicine, London, UK

\subsection{6/jech-2018-SSMabstracts.131}

Background Studies of neighbourhood built environments and obesity-related outcomes have produced inconsistent findings in different settings. One explanation for this may be that built environment effects on health are context dependent, and therefore vary geographically. Understanding broader contextual factors that might modify or influence health effects of neighbourhood built environments could help identify conditions under which neighbourhood interventions are more likely to succeed. Using the large, geographically diverse UK Biobank sample and linking it to other area-level data, we examine whether various contextual factors at multiple scales modify our previously observed associations between the neighbourhood physical activity (PA) environment and adiposity, and/or predict geographical heterogeneity of those associations.

Methods The UK Biobank cohort comprises approximately 400000 adults aged 40-69, recruited from across the UK between 2006 and 2010 using a clustered sampling design. Linked to each individual is detailed information about their neighbourhood environment, derived from national spatial databases. First, we examine whether cross-sectional associations between the number of formal PA facilities within $1 \mathrm{~km}$ of people's homes, and BMI, are modified by other neighbourhood characteristics (e.g. greenspace) operating at the same scale, by fitting interaction terms between the PA environment and potential modifiers and examining stratum-specific associations. Second, we describe how the main association varies geographically across UK nations, regions and local authorities, then explore how contextual factors at various scales might explain this variation. We apply single and multilevel regression modelling techniques to a dataset we have constructed by mapping the UK Biobank sample and linking it to publicly available data on a range of geodemographic and environmental characteristics of areas.

Results While the overall association between the PA environment and BMI is negative, models stratified by other neighbourhood characteristics showed some evidence of effect modification at this scale. The main association also varied geographically at various scales, even after comprehensive adjustment for sociodemographic and other characteristics of individuals. For example, we observed an association of above-average magnitude in Scotland, but below-average in Wales, and strong in Bristol and Glasgow, but null in parts of Yorkshire and the North East. Preliminary results suggest characteristics of the broader areas in which neighbourhoods are located may explain some of this observed variation.

Conclusion Associations between neighbourhood PA environments and BMI appear to vary across the UK, at multiple geographical scales. Understanding this heterogeneity may help identify where built environment interventions might be expected to succeed or fail, and what contextual factors might support such interventions.

\section{P6 VARYING MENTAL HEALTH IN THE POPULATION ACROSS SCOTLAND DURING THE RECENT RECESSION}

${ }^{1} \mathrm{M}$ Cherrie*, ${ }^{1} \mathrm{~J}$ Pearce, ${ }^{1,2} \mathrm{~S}$ Curtis, ${ }^{1,3} \mathrm{C}$ Dibben, ${ }^{2} \mathrm{~N}$ Cunningham, ${ }^{4} \mathrm{C}$ Bambra. ${ }^{1} \mathrm{C}$ entre for Research on Environment, Society and Health, University of Edinburgh, Edinburgh, UK; ${ }^{2}$ Geography, Durham University, Durham, UK; ${ }^{3}$ ESRC Administrative Data Research Centre, University of Edinburgh, Edinburgh, UK; ${ }^{4}$ Medical School, Newcastle University, Newcastle upon Tyne, UK

\subsection{6/jech-2018-SSMabstracts. 132}

Background This research focusses on geographical variation in population mental health over the period 2007-2011 (during the onset of the economic recession). We report preliminary results from a project recently funded under the ESRC SDAI programme, seeking to explore variability in mental illness in Scotland during this period. Our methods combine information on individual lifecourse changes, as well as change over time in areas where the individuals are living. This research contributes to a growing field concerned with the relationships between population health and changes in wider determinants of health, operating over time for both individual people and places where they live.

Methods We are making innovative use of a variety of data sources including individual data from the Scottish Longitudinal Study (SLS), a large (5\%) sample from the Scottish population; drawn from the population census made available under secure conditions at the Longitudinal Studies Centre Scotland, with help and supervision of SLS staff. (SLS is supported by the ESRC/JISC, the Scottish Funding Council, the Scientists Office and the Scottish Government.) We report on work which has linked these data to information on prescriptions likely to be used to treat mental illness (provided by the Electronic Data Research \& Innovation Service (eDRIS) and information on area socio-economic conditions publicly available via Scottish National Government and NOMIS (Durham University) a service provided by the Office for National Statistics, ONS.

Results We report preliminary results from a dataset for more than 120000 people. Most of those reporting mental illness were taking antidepressants. There is a significant statistical 
association between risk of reporting mental illness in 2011 and employment trajectory of local authority of residence by 2011, (after controlling for individual risk factors and for neighbourhood deprivation in 2001, before the onset of the recession).

Conclusion Various personal, family and neighbourhood factors are associated with self-reported mental illness. Allowing for individual/family factors and local deprivation, people in local authorities where employment rates remained higher during the recession had lower risk of reporting mental illness, especially in the highlands and Islands of Scotland. Further research is being carried out to explore these relationships (eg controlling for migration and other possible area level determinants of mental health). The research underlines the importance of maintaining mental health services across Scotland during the recession to protect mental health and control inequality.

\section{\begin{tabular}{lll}
\hline P7 THE IMPACT OF REGIONAL EMPLOYMENT LEVELS \\
\hline
\end{tabular} DURING THE GREAT RECESSION (2008 TO 2013) AND WORKLESSNESS ON THE HEALTH OF THE WORKING- AGE POPULATION: CROSS-NATIONAL ANALYSIS OF 16 EUROPEAN COUNTRIES}

${ }^{1} \mathrm{C}$ Niedzwiedz*, ${ }^{2} \mathrm{~K}$ Thomson, ${ }^{2} \mathrm{C}$ Bambra, ${ }^{3} \mathrm{~J}$ Pearce. ${ }^{1}$ Institute of Health and Wellbeing, University of Glasgow, Glasgow, UK; ${ }^{2}$ Institute of Health and Society, Newcastle University, Newcastle upon Tyne, UK; ${ }^{3}$ Centre for Research on Environment, Society and Health, University of Edinburgh, Edinburgh, UK

\subsection{6/jech-2018-SSMabstracts. 133}

Background Studies from single countries suggest that local labour market conditions, including rates of employment, tend to be associated with the health of the populations residing in those areas, even after adjustment for individual characteristics including employment status. The aim of this study is to strengthen the cross-national evidence base on the influence of regional employment levels and individual worklessness on health. Our objectives are to investigate whether higher regional employment levels are associated with better health over and above individual-level employment. This could be due to pathways such as weakening community cohesion, increasing place-based stigma and declining regional income.

Methods Individual-level data ( $n=20485$ aged 15 to 64 years) were taken from 16 countries in the European Social Survey $(2014 / 15)$ and regional employment rates extracted from Eurostat. Health outcomes included self-reported heart or circulation problems, high blood pressure, self-rated health, depressive symptoms, obesity and allergies (as a falsification test). Our exposures of interest included worklessness, defined as individuals who did not report being in paid work. At the regional level we included the average employment rate of those aged 15 to 64 years from 2008 to 2013. We calculated multilevel Poisson regression models for the binary outcomes (calculating the incidence rate ratio (IRR)), which included individuals nested within NUTS regions and linear multilevel regression models for continuous measures, controlling for potential confounding variables.

Results Between 2008 and 2013 the employment rate declined the most in Spain and increased the most in Germany. Increased average regional employment rates were associated with better health outcomes: heart/circulation problems $\mathrm{IRR}=0.970 \quad(95 \%$ CI 0.950 to 0.990$)$; high blood pressure
IRR $=0.981$ (95\% CI 0.965 to 0.997$)$; poor self-rated health $\mathrm{IRR}=0.974 \quad(95 \%$ CI 0.956 to 0.992$)$; obesity $\mathrm{IRR}=0.971$ (95\% CI 0.960 to 0.982 ); depressive symptoms $b=0.992$ (95\% CI 0.987 to 0.997$)$, allergies $\mathrm{IRR}=0.995$ (0.977 to 1.013). Individual worklessness was associated with all health outcomes, most strongly with poor self-rated health. In models including both individual worklessness and the average regional employment rate, the latter remained associated only for obesity.

Discussion Lower regional employment levels and worklessness are associated with adverse health outcomes across European countries. When accounting for both individual- and regionallevel employment variables, a separate association between the regional employment level was suggested for obesity. The key strength of our study was the use of comparable cross-national data that integrated individual- and regional-level variables, but is limited by the use of self-reported data. Further exploration of potential causal mechanisms is needed.

\section{P8 INVESTIGATING EPIGENETIC DIFFERENCES IN RESPONSE TO SHIFT WORK: FINDINGS FROM UNDERSTANDING SOCIETY (UK LONGITUDINAL HOUSEHOLD SURVEY)}

${ }^{1}$ RC Richmond*, ${ }^{2} Y$ Bao, ${ }^{2} \mathrm{M}$ Smart, ${ }^{3} \mathrm{~T}$ Gorrie-Stone, ${ }^{3} \mathrm{~L}$ Schalkwyk, ${ }^{4} \mathrm{~J}$ Mill, ${ }^{1} \mathrm{G}$ Davey Smith, ${ }^{2} \mathrm{M}$ Benzeval, ${ }^{1} \mathrm{C}$ Relton, ${ }^{2} \mathrm{M}$ Kumari. ${ }^{1} \mathrm{MRC}$ Integrative Epidemiology Unit, Bristol Medical School, University of Bristol, Bristol, UK; ${ }^{2}$ Institute of Social and Economic Research, University of Essex, Colchester, UK; ${ }^{3}$ School of Biological Sciences, University of Essex, Colchester, UK; ${ }^{4}$ University of Exeter Medical School, University of Exeter, Exeter, UK

\subsection{6/jech-2018-SSMabstracts. 134}

Background Shift work is a feature of many occupations and has been associated with a range of adverse health outcomes, including obesity, diabetes, depression and cancer. The main proposed mechanism linking them is a disruption in circadian rhythms, particularly among night shift workers. DNA methylation may serve as a biomarker for circadian disruption and a potential mechanism by which shift work influences disease risk. In the context of a longitudinal study, we aimed to investigate whether shift work is associated with DNA methylation.

Methods Methylation profiling was performed using Illumina EPIC micro-arrays on whole-blood DNA samples, obtained from British Household Panel Survey (BHPS) participants of Understanding Society from 2010-2012. BHPS comprises a clustered random sample of households recruited in 1991, with all members followed annually. After pre-processing, 1175 samples and 857,071 CpG sites remained for investigation. Shift work variables were derived from 17 time points between 1991 and 2009: ever $(n=359)$, current $(n=88)$ and long-term ( ${ }^{3} 3$ years) shift work $(n=154)$ (night and rotating). Epigenome-wide association analysis was performed using multivariable regression with adjustment for age, sex, batch and blood processing day. Further models were adjusted for celltype composition and socio-economic variables. Methylation age was also estimated based on the Horvath epigenetic clock and the impact of shift work on 'epigenetic age acceleration' (EAA) was investigated.

Results In epigenome-wide association analysis, $50 \mathrm{CpG}$ sites were associated with shift work with a nominal p-value $<1 \times$ $10^{-5}$ across the 9 main analyses, with the strongest signal of association at $\operatorname{cg} 12880856$ (PPARG) identified in relation to 